\title{
A Collective Extension of Relational Grammar
}

\author{
MICHAEL BÖTTNER, Max-Planck-Institut für Psycholinguistik, \\ Nijmegen, The Netherlands, E-mail: boettner@mpi.nl.
}

\begin{abstract}
Relational grammar was proposed in Suppes (1976) as a semantical grammar for natural language. Fragments considered so far are restricted to distributive notions. In this article, relational grammar is extended to collective notions.
\end{abstract}

Keywords: Collectivity, distributivity, relational algebra, Peirce algebra, relational grammar, algebraic semantics, variablefree semantics.

\section{Introduction}

The notion of a relational grammar was proposed. by Suppes as a grammar with a model-theoretic semantics for natural languages. ${ }^{\eta_{1}^{\prime}}$ It maps natural language expressions onto terms of relational algebra. The construction of algebraic terms from constants by operations fits the constituent structure of a natural language expression much better than conventional quantifier variable structures. Being rich enough to express classical mathematics, powerful and elegant tool for natural language semantics.

Relational grammars so far have been presented mostly for English fragments: A fragment for sentences occurring in the syllogism was given in Suppes (1976). The fragment was extended to attributive constructions and possessive NP constructions in Suppes and Macken (1978) and to intonation in Suppes (1979). A fragment for English imperatives was presented in Böttner (1992a). An analysis of copulative terms is given in Böttner (1994). In Böttner (1992b) a proposal was made for the analysis of English constructions with anaphorical pronouns. No precise rules of grammar had been given. The purpose of this paper is to provide a relational grammar for the constructions in question. Whereas in the earlier paper the thrust was on a variablefree semantics for certain anaphoric structures, the main result of this paper is that all the structures mentioned in Böttner (1992b) can indeed be accommodated in a relational grammar.

All the notions considered in various fragments satisfy the following property: if they hold of a class of individuals they also hold of each individual of that class. Notions of this kind are called distributive. It is well-known that not all natural language notions are of that kind. An example is John and Mary are a couple. Verb phrases of this kind are called collective. In this paper, an extension of relational grammar for collectives will be proposed and extended to anaphora.

\footnotetext{
${ }^{1}$ Suppes (1976).

${ }^{2}$ Tarski and Givant (1987).

L. J. of the IGPL, Vol. 6 No. 2, pp.

(C) Oxford University Press
} 
Our procedure will be as follows: We start from the calculus of sets and relations. On the basis of standard operations we define some new operations. We then show the usefulness of these operations for the analysis of certain English constructions, in particular for collective verb phrases and certain anaphoric constructions that go with them. The notions from the calculus of sets and binary relations are introduced in 1 . The notion of a relational grammar is illustrated by some example grammar in 2. In 3 this grammar is extended to capture collective notions. In 4 the grammar is extended to capture some constructions with anaphoric pronouns. In a final section 5 , some problems of the analysis are pointed out.

\section{Calculus of Sets and Binary Relations}

All we presuppose for the following is elementary set theory. Let us assume some set $D$ and consider subsets of $D$ and binary relations on $D$. I assume familiarity with the operations of set theory like intersection, union, complement, or difference. We use $\emptyset$ for both the empty set and the empty relation, $I$ for the identity relation on $D$. We use the conversion of a relation $R$ defined

$$
\breve{R}=\{<x, y>\mid<y, x>\in R\},
$$

and composition of two binary relations $R$ and $S$ defined

$$
R ; S=\{<x, y>\mid(\exists z)(x R z \wedge z S y)\} .
$$

There are two variants of the composition operation depending on whether one of the arguments is a subset of $D$. If the first argument of composition is a subset of $D$, this leads to the operation of upper image of a set $A$ under a relation $R$ defined

$$
R " A=\{y \mid(\exists x)(x \in A \wedge x R y)\} .
$$

Familiar from set theory is also the operation of the Cartesian Product of sets $A$ and $B$ :

$$
A \times B=\{<x, y>\mid x \in A \wedge y \in B\} .
$$

From the elementary theory of binary relations the following theorems are known to hold:

TheOREM 2.1

1. $A \times-B \subseteq-(A \times B)$

2. $R ;(S ; T)=(R ; S) ; T$

3. $(R, S)^{\sqcup}=\breve{S} ; \breve{R}$

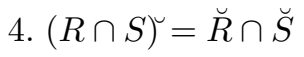

5. $(A \times B) \cap R \neq \emptyset \leftrightarrow A \cap\left(\breve{R}^{\prime} B\right) \neq \emptyset$

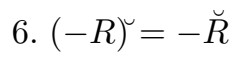


From our list of primitive operations many more operations can be defined, like, for instance:

$$
\begin{aligned}
\operatorname{dom}(R) & =\breve{R} \text { “ } D \\
E(A) & =\operatorname{dom}(D \times A) \\
U(A) & =-E(-A) \\
N(A) & =-E(A) \\
e(R, A) & =\breve{R} \text { “A } \\
u(R, A) & =-e(-R, A) \\
\operatorname{Refl}(R) & =\operatorname{dom}(R \cap I) \\
I_{A} & =I \cap(A \times A) \\
\operatorname{Poss}(A) & =P \text { “ } \\
\operatorname{Poss}_{(R, A)} & =-\operatorname{dom}\left(\left(P ; I_{A}\right) \cap-R\right) \\
\operatorname{Col}_{e}(R, A) & =R ; I_{A} ; \breve{R} \\
\operatorname{Col}_{u}(R, A) & =u(R, A) \times u(R, A) \\
\operatorname{sym}(R) & =R \cap \breve{R} \\
\operatorname{Rec}(R) & =\operatorname{sym}(R)-I \\
\operatorname{RecPoss}(R, A) & =\operatorname{sym}\left(-\left(-R ; I_{A} ; \breve{P}\right)\right)-I \\
\operatorname{Id}_{(}(R, A) & =-\left(R ; I_{A} ;-\breve{R}\right) \cap-\left(-R ; I_{A} ; \breve{R}\right) \\
\operatorname{Div}(R, A) & =-\left(R ; I_{A} ; \breve{R}\right) \cap-\left(-R ; I_{A} ;-\breve{R}\right)
\end{aligned}
$$

The operations dom, E, $U, N, e, u, R e f l$ and Poss return a set, the other operations return a binary relation. More specifically, dom returns the domain of its argument relation. The operation $e(R, A)$ is called the upper counterimage of set $A$-under relation $R$, also known by the name of Peirce Product of relation $R$ to set $A .{ }^{3}$ The operation $u(R, A)$ had already been used by de Morgan and is known under the term of ordinary involution. ${ }^{4} I_{A}$ is the identity relation over set $A$.5 The operation $\operatorname{sym}(R)$ returns the greatest symmetric subrelation of its argument relation $R$. In the definition of Poss a relation $P$ is used that does not occur in the parameters of the operation Poss, since we think of $P$ as a constant denoting the relation of possession in all models! $!_{1}^{1}$

THEOREM 2.2

$$
E(A)= \begin{cases}D & \text { if } A \neq \emptyset \\ \emptyset & \text { otherwise }\end{cases}
$$

Proof. i. $A \neq \emptyset \Rightarrow \operatorname{dom}(D \times A)=D$.

ii. $A=\emptyset \Rightarrow \operatorname{dom}(D \times A)=\operatorname{dom}(D \times \emptyset)=\operatorname{dom}(\emptyset)=\emptyset$. Q.E.D.

$E$ is a two-valued operation. It returns $D$ in case its argument is not the empty set, and returns the empty set otherwise.

From elementary set theory we have the following theorem:

\footnotetext{
${ }^{3}$ cf. Brink (1981).

${ }^{4}$ cf. Brink (1978).

${ }^{5}$ cf. Suppes (1960).

${ }^{6}$ This was suggested in Suppes and Macken (1978).
} 
178 A Collective Extension of Relational Grammar

THEOREM 2.3

$A \neq \emptyset \rightarrow u(R, A) \subseteq e(R, A)$.

A binary relation $R$ is called symmetrical if $\breve{R} \subseteq R$.

THEOREM 2.4

$\operatorname{sym}(R)$ is symmetrical.

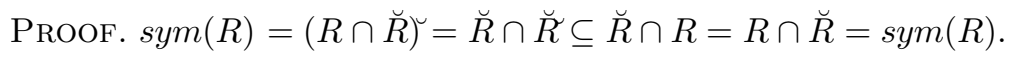

THEOREM 2.5

$I$ is symmetrical.

THEOREM 2.6

If $R$ is symmetrical and $S$ is symmetrical, then $R \cap S$ is symmetrical.

Proof. Follows from definition and Theorem $2.2 i$

THEOREM 2.7

The relation $\operatorname{Rec}(\mathrm{R})$ is symmetrical.

Proof. Follows from Theorem $2 . \overline{2}, 2.5$, and 2.6 .

THEOREM 2.8

$\operatorname{RecPoss}(R)$ is symmetrical.

Proof. Follows from Theorem $2 . \overline{2}, \overline{2} \cdot \overline{5}$, and 2.6.

THEOREM 2.9

$\operatorname{Id}(R, A)$ is symmetrical.

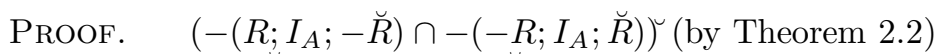

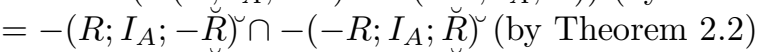

$$
\begin{aligned}
& =-\left(-R ; I_{A} ; \breve{R}\right) \cap-\left(R ; I_{A} ;-\breve{R}\right)(\text { by Theorem } 1 \text {, iii) } \\
& =-\left(R ; I_{A} ;-\breve{R}\right) \cap-\left(-R ; I_{A} ; \breve{R}\right) \text {. }
\end{aligned}
$$

A binary relation $R$ is called transitive if $R ; R \subseteq R$.

THEOREM 2.10

$\operatorname{Id}(R, A)$ is transitive.

Proof. $\langle x, y>\in I d(R, A)$, i.e. $(\forall u)(u \in A \rightarrow(x R u \leftrightarrow y R u)$ (premise 1)

$<y, z>\in I d(R, A)$, i.e. $(\forall u)(u \in A \rightarrow(y R u \leftrightarrow z R u)$ (premise 2)

Let $u \in A$. Then $x R u \leftrightarrow y R u$ (from premise 1) and $y R u \leftrightarrow z R u$ (from premise 2), hence, $x R u \leftrightarrow z R u$, i.e. $\langle x, z>\in \operatorname{Id}(R, A)$.

$R$ is an equivalence relation iff $R$ is transitive and symmetrical.

THEOREM 2.11

The relation $\operatorname{Id}(R, A)$ is an equivalence relation.

Proof. Follows from Theorem 2.9 and Theorem $2 . \overline{1}$

THEOREM 2.12

$\operatorname{Div}(R, A)=-\operatorname{Id}(R, A)$. 
Proof. We show

1. $\operatorname{Div}(R, A) \subseteq-\operatorname{Id}(R, A)$

2. $-\operatorname{Div}(R, A) \subseteq \operatorname{Id}(R, A)$

1. $\operatorname{Div}(R, A) \cap \operatorname{Id}(R, A)$

$=-\left(R ; I_{A} ; \breve{R}\right) \cap-\left(-R ; I_{A} ;-\breve{R}\right) \cap-\left(R ; I_{A} ;-\breve{R}\right) \cap-\left(-R ; I_{A} ; \breve{R}\right)$

$=-\left(R ; I_{A} ; \breve{R}\right) \cap-\left(R ; I_{A} ;-\breve{R}\right) \cap-\left(-R ; I_{A} ;-\breve{R}\right) \cap-\left(-R ; I_{A} ; \breve{R}\right)$

$=-\left(\left(R ; I_{A} \breve{R}\right) \cup\left(R ; I_{A} ;-\breve{R}\right) \cap-\left(\left(-R ; I_{A} ;-\breve{R}\right) \cup\left(-R ; I_{A} ; \breve{R}\right)\right)\right.$

$=-\left(\left(R ; I_{A} ;(\breve{R} \cup-\breve{R})\right) \cap-\left(-R ; I_{A} ;(-\breve{R} \cup \breve{R})\right)\right)$

$=-\left(R ; I_{A} ;(D \times D)\right) \cap-\left(-R ; I_{A} ;(D \times D)\right)$

$=-\left(\left(R ; I_{A} ; D \times D\right) \cup\left(-R ; I_{A} ;(D \times D)\right)\right)$

$=-\left((R \cup-R) ; I_{A} ;(D \times D)\right)$

$=-\left((D \times D) ; I_{A} ;(D \times D)\right)$

$=-(D \times D)$

$=\emptyset$

2. $\quad-\operatorname{Div}(R, A) \cap-I d(R, A)$

$=-\left(R ; I_{A} ; \breve{R}\right) \cap-\left(-R ; I_{A} ;-\breve{R}\right) \cap-\left(R ; I_{A} ;-\breve{R}\right) \cap-\left(-R ; I_{A} ; \breve{R}\right)$

$=-\left(R ; I_{A} ; \breve{R}\right) \cap-\left(R ; I_{A} ;-\breve{R}\right) \cap-\left(-R ; I_{A} ;-\breve{R}\right) \cap-\left(-R ; I_{A} ; \breve{R}\right)$

$=-\left(\left(R ; I_{A} ; \breve{R}\right) \cup\left(R ; I_{A} ;-\breve{R}\right)\right) \cap-\left(\left(-R ; I_{A} ;-\breve{R}\right) \cup\left(-R ; I_{A} ; \breve{R}\right)\right)$

$=-\left(R ; I_{A} ;(\breve{R} \cup-\breve{R})\right) \cap-\left(-R ; I_{A} ;(-\breve{R} \cup \breve{R})\right)$

$=-\left(R ; I_{A} ;(D \times D)\right) \cap-\left(-R ; I_{A} ;(D \times D)\right)$

$=-\left(\left(R ; I_{A} ;(D \times D)\right) \cup\left(-R ; I_{A} ;(D \times D)\right)\right)$

$=-\left((R \cup-R) ; I_{A} ;(D \times D)\right)$

$=-\left((D \times D) ; I_{A} ;(D \times D)\right)$

$=-(D \times D)$

$=\emptyset$.

THEOREM 2.13

$R ; I_{A} ; \breve{R} \subseteq e(R, A) \times e(R, A)$.

$\begin{aligned} R ; I_{A} ; \breve{R} & =R ; I_{A} ; I_{A} ; \breve{R} \\ \text { Proof. } & \subseteq \operatorname{dom}\left(R ; I_{A}\right) \times \operatorname{dom}\left(\left(I_{A} ; \breve{R}\right)^{y}\right)\end{aligned}$

$=\operatorname{dom}\left(R ; I_{A}\right) \times \operatorname{dom}\left(R ; I_{A}\right)$

$=e(R, A) \times e(R, A)$ since $R ; S \subseteq \operatorname{dom}(R) \times \operatorname{dom}(\breve{S})$

THEOREM 2.14

$\mathrm{Col}_{u}(R, A) \subseteq I d(R, A)$.

Proof. 1. Let $A=\emptyset$. Then $\operatorname{Col}_{u}(R, A)=D \times D$ and $\operatorname{Id}(R, A)=D \times D$.

Hence $\operatorname{Col}_{u}(R, A)=\operatorname{Id}(R, A)$.

2. Let $A \neq \emptyset$. Therefore $u(R, A) \subseteq e(R, A)$. From this we get:

(a) $u(R, A) \times u(R, A)$

$$
\begin{aligned}
& =-e(-R, A) \times e(R, A) \text { (by Definition of } E \text { ) } \\
& \subseteq-e(-R, A) \times e(R, A) \text { (by Theorem } 2.3 i \text { ) } \\
& \subseteq-(e(-R, A) \times e(R, A)) \text { (by Theorem } 2.2 i) \\
& \left.=-\left(-R ; I_{A} ; \breve{R}\right) \text { (by Theorem } \underline{2} . \overline{1} \underline{j}^{i}\right)
\end{aligned}
$$


(b) $u(R, A) \times u(R, A)$

$\subseteq e(R, A) \times u(R, A)$ (by Theorem 2.3$)$

$=e(R, A) \times-e(-R, A)$ (by Definition of $E)$

$\subseteq-(e(R, A) \times e(-R, A)$ (by Theorem $\overline{2} .2 i)$

$\subseteq-\left(R ; I_{A} ;-\breve{R}\right)$ (by Theorem $\dot{2} . \overline{1} \bar{i}$ )

Since $C_{0} l_{u}(R, A)=u(R, A) \times u(R, A)$,

and $I d(R, A)=-\left(R ; I_{A} ;-\breve{R}\right) \cap-\left(-R ; I_{A} ; \breve{R}\right)$,

assertion follows.

In our calculations we have made use of laws from the theory of sets and binary relations. The laws of set-theory are captured under the notion of a Boolean algebra. Many laws referring to binary relations can be captured under the notion of a relation algebra. An equational definition of relation algebra was first given by Tarski. ? $^{7}$ An equational definition of the underlying structure was given under the notion of a Peirce algebra.8

\section{Relational Grammar}

A relational grammar is a denoting grammar. A denoting grammar is a context-free grammar with a model-theoretic semantics. . $^{2}$ With each production rule, a semantic function is associated. The characteristic feature of a relational grammar is that its denotations are restricted to subsets of the domain $D$ and to binary relations on $D$, i.e. to the hierarchy

$$
\mathcal{H}(D)=\mathcal{P}(D) \cup \mathcal{P}(D \times D)
$$

where $D$ is some non-empty set.10

$$
10
$$

An example of a denoting grammar is the following:

$$
\begin{aligned}
S & \rightarrow P N+V P \\
S & \rightarrow U Q+N+V P \\
S & \rightarrow E Q+N+V P \\
S & \rightarrow N Q+N+V P \\
S & \rightarrow C P N P+V P \\
C P N P & \rightarrow P N+C C+P N^{\prime} \\
C P N P & \rightarrow P N+C P N P \\
N P & \rightarrow P N+\text { PossInf }+R N \\
N P & \rightarrow P N+P o s s I n f+N \\
V P & \rightarrow C o p+N P \\
V P & \rightarrow T V+P N \\
V P & \rightarrow T V+U Q+N \\
V P & \rightarrow T V+E Q+N \\
V P & \rightarrow T V+R e f l \\
V P & \rightarrow T V+P o s s+N \\
V P & \rightarrow T V+C P N P
\end{aligned}
$$$$
[S]=E([P N] \cap[V P])
$$$$
[S]=U(-[N] \cup[V P])
$$$$
[S]=E([N] \cap[V P])
$$$$
[S]=N([N] \cap[V P])
$$$$
[S]=U(-[C P N P] \cup[V P])
$$$$
[C P N P]=[P N] \cup\left[P N^{\prime}\right]
$$$$
[C P N P]=[P N] \cup[C P N P]
$$$$
[N P]=e([R N],[P N])
$$$$
[N P]=\operatorname{Poss}([P N]) \cap[N]
$$$$
[V P]=[N P]
$$$$
[V P]=e([V P],[P N])
$$$$
[V P]=u([T V],[N])
$$$$
[V P]=e([T V],[N])
$$$$
[V P]=\operatorname{Refl}([T V])
$$$$
[V P]=\operatorname{Poss}([T V],[N])
$$$$
[V P]=U([T V],[C P N P])
$$

\footnotetext{
${ }^{7}$ Tarski (1941).

${ }^{8}$ Brink, Britz, and Schmidt (1994).

${ }^{9}$ Suppes (1973).

${ }^{10}$ Suppes (1976).
} 
where S (sentence), NP (noun phrase), VP (verb phrase), and CPNP (conjoined proper noun phrase) refer to phrasal categories. We distinguish the following lexical categories:

$\begin{array}{lll}\text { EQ } & \text { existential quantifier } & \text { some } \\ \text { UQ } & \text { universal quantifier } & \text { all } \\ \text { NQ } & \text { negative quantifier } & \text { no } \\ \text { PN } & \text { proper noun } & \text { John, Mary, Bill, Dick, Harry } \\ \text { N } & \text { common noun } & \text { books, houses, toys, students, guests } \\ \text { RN } & \text { relational noun } & \text { brother, brothers } \\ \text { TV } & \text { transitive verb } & \text { own, like } \\ \text { CC } & \text { conjoining conjunction } & \text { and } \\ \text { Cop } & \text { copula } & \text { is, are } \\ \text { Refl } & \text { reflexive pronoun } & \text { himself, herself, themselves } \\ \text { Poss } & \text { possessive pronoun } & \text { his, her, their } \\ \text { PossInf } & \text { possessive inflexion } & \text { 's }\end{array}$

The notion of a denoting grammar gives rise to the notion of a semantic tree. A semantic tree of a denoting grammar $G$ is a derivation tree of the context-free grammar $G$ with the root node having a denotation that is derived from the denotations of its daughter nodes by semantic functions. The leaves of the semantic tree get their denotation (in some model) by a function into the hierarchy $\mathcal{H}(D)$. For purposes of illustration let us give a couple of examples of semantic trees that can be derived in our grammar.

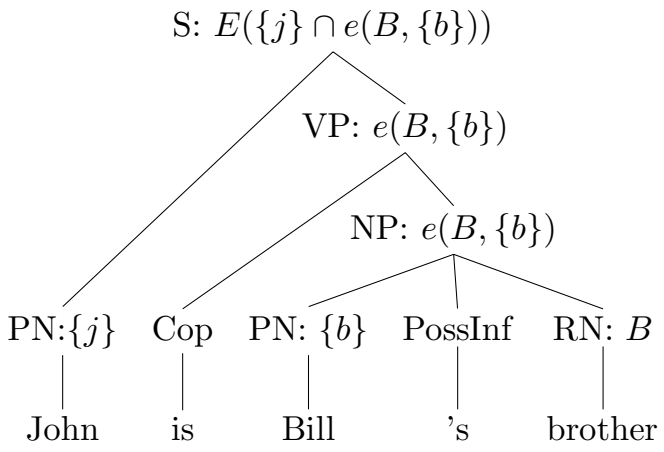

Recall from Theorem 2.2 that $E$ is a two-valued operation. We can identify the two values with the truth values: A sentence is called true with respect to a model structure if the root node denotation is $D$ otherwise false. 


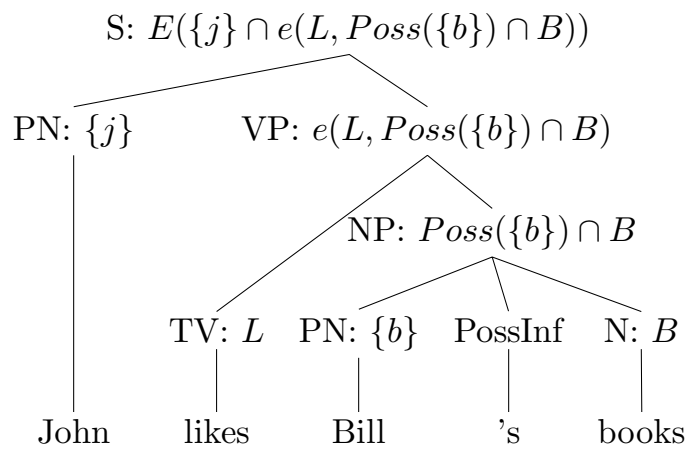

Our solution for Bill's books is in line with the solution proposed by Suppes and Macken.' In the case of Bill's brother that would be treated similarly by Suppes and Macken, our solution does not use the possessive relation $P$. We think that the words books and brother belong to different categories: books belongs to the category $N$ of a classificatory common noun, brother belongs to the category $R N$ of a relational common noun.

Our grammar derives the following constructions with proper noun coordinations:

$$
\begin{array}{ll}
\text { John and Mary are students } & U(-(\{j\} \cup\{m\}) \cup S) \\
\text { like John and Mary } & u(L,\{j\} \cup\{m\})
\end{array}
$$

where John denotes $\{j\}$, Mary denotes $\{m\}$ and $S$ is the set of students and $L$ is the relation denoted by the verb like.

Notice that the language generated by these rules is not restricted to just two constituents but can derive copulative terms with any number of constituents.
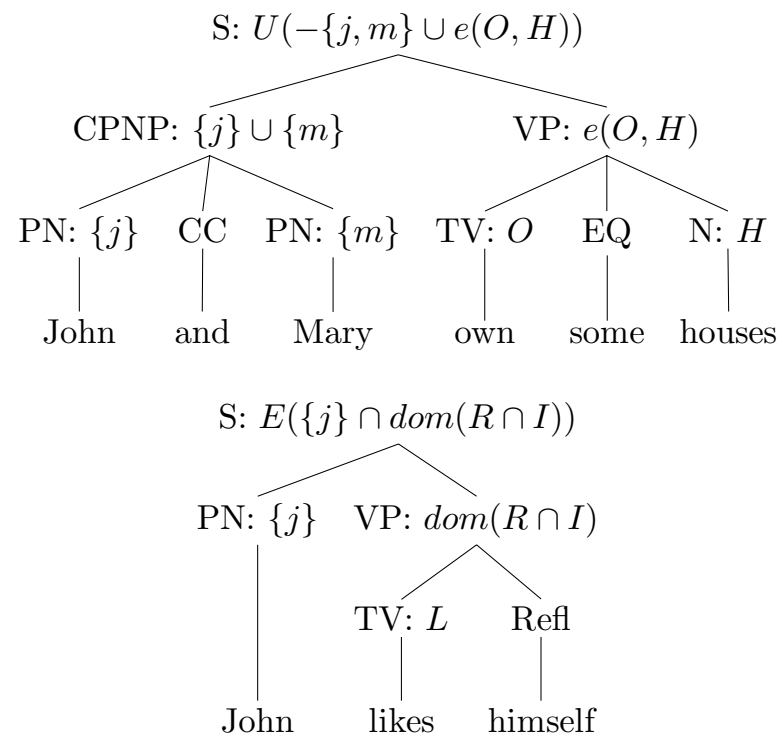

\footnotetext{
${ }^{11}$ Suppes and Macken (1978).
} 


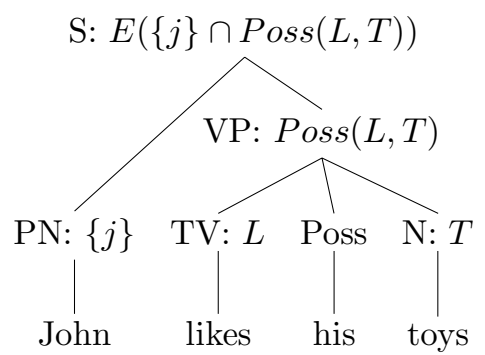

According to our grammar, the phrase likes his toys denotes the term

$$
-\operatorname{dom}\left(\left(P ; I_{T}\right) \cap-L\right) \text {. }
$$

That (3.7i) corresponds to the intuitive meaning of like his toys follows from the fact that $(3.7)$ is equivalent to the quantifier expression

$$
\{x \in D:(\forall y)(y \in T \wedge x P y \rightarrow x L y)\} .
$$

\section{Collective Extension}

There are many constructions that cannot be analyzed with our grammar developed so far. Our focus here will be on constructions like the following one:

$$
\text { John and Bill are brothers }
$$

Although the structure of (4.1.1) closely resembles the structure of

$$
\text { John and Mary are students }
$$

its meaning is equivalent to that of (3.2i).

It has been proposed that denotations for collective predicates should be sets of sets of objects.12! Within the confines of our set-theoretical hierarchy $\mathcal{H}(D)$ we cannot have properties of that kind. For any such property would be a set $X$ of elements of $\mathcal{P}\left(D_{\vec{r}}\right.$, i.e. $X \in \mathcal{P}(\mathcal{P}(D))$. I propose to construe collective predicates as binary relations.1.Notice that our analysis of collectives stays within the confines of the restricted settheoretical hierarchy $\mathcal{H}(D)$. This makes jt, different from proposals along the lines of

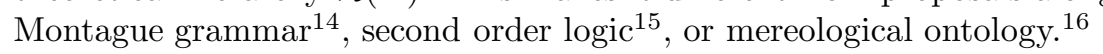

For the derivation of collectives our grammar is extended by the following rules:

$$
\begin{aligned}
& S \rightarrow P P N P+\operatorname{ColVP} \quad[S]=E([P P N P] \cap([\text { ColVP }])) \\
& S \rightarrow U Q+N+\operatorname{ColvP} \quad[S]=U(-([N] \times[N]-I) \cup[\text { ColvP }]) \\
& S \rightarrow E Q+N+\operatorname{ColVP} \quad[S]=E([N] \times[N] \cap[\text { ColvP }]) \\
& P P N P \rightarrow P N+C C+P N^{\prime} \quad[P P N P]=[P N] \times\left[P N^{\prime}\right] \\
& \text { ColvP } \rightarrow \text { Cop }+R N \quad[\text { ColVP }]=[R N] \\
& \operatorname{ColVP} \rightarrow T V+E Q+N \quad \operatorname{ColVP}=\operatorname{Col}_{E}([T V],[N]) \\
& \mathrm{ColVP} \rightarrow T V+U Q+N \quad \operatorname{ColVP}=\operatorname{Col}_{U}([T V],[N])
\end{aligned}
$$

\footnotetext{
${ }^{12}$ Hausser (1974), Hoeksema (1988), Keenan and Faltz (1985).

${ }^{13}$ This proposal can be traced back to Peirce (1880).

${ }^{14}$ Hausser (1974).

${ }^{15}$ Blau (1981).

${ }^{16}$ Link (1983).
} 
where ColVP $=$ collective verb phrase and PPNP $=$ paired proper noun phrase.

We therefore have the following semantic tree for $\left(4 . \mathbf{l}_{-1}\right)$ :

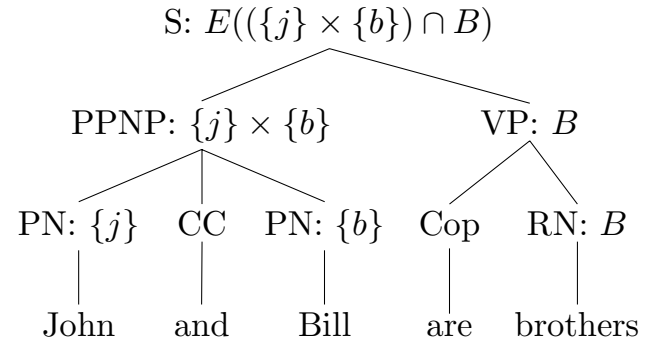

We are now able to prove that $(\overline{4} . \overline{1})$ is equivalent is to $(\overline{3} . \overline{2} \cdot \overline{1})$ :

$$
\begin{aligned}
E(\{j\} \cap e(B,\{b\})=D & \leftrightarrow\{j\} \cap \breve{B} "\{b\} \neq \emptyset \text { (Definition of } E) \\
& \leftrightarrow\{j\} \times\{b\} \cap B \neq \emptyset \text { (by Theorem 2.1,v) } \\
& \leftrightarrow E(\{j\} \times\{b\} \cap B) \text { (Definition of } E \text { ) }
\end{aligned}
$$

The semantic function for the rule

$$
S \rightarrow U Q+N+\operatorname{ColVP}
$$

may be surprising for the reason that it explicitly excludes identical pairs. The reason for doing so is that we want the sentence

$$
\text { All guests are brothers }
$$

to be true in a model structure

$$
\begin{aligned}
& G=\{a, b, c\} \\
& B=\{\langle a, b\rangle,\langle a, c\rangle,\langle b, c\rangle,\langle b, a\rangle,\langle c, a\rangle,\langle c, b\rangle\}
\end{aligned}
$$

where set $G$ is the denotation of guests and binary relation $B$ is the denotation of brother. Without explicitly excluding $I$ from $G \times G$, however, the sentence will be false, since $<a, a>\notin B$ for the relation denoted by brother is conventionally assumed to be irreflexive.

In departure from our general convention, the semantic function $\operatorname{Col}_{u}(R, A)$ is not defined in line with the general transformation:

$$
U(\ldots)=-E(-\ldots)
$$

as one would expect. Applying this transformation to collective verb phrases would leave us with the relation

$$
\{<x, y>\mid(\forall z)(z \in A \rightarrow(x R z \vee y R z))\}
$$

as the denotation for the phrase own all houses. We think that this is not strong enough to account for the meaning of this phrase.

Some verb phrases can be used both distributively and collectively. Consider, for instance, the predicate denoted by own a house. In John owns a house it can only be used distributively. In John and Mary own a house it can be used both distributively 
and collectively. The distributive reading admits of the possibility that there are two houses: one belonging to John, the other belonging to Mary. The collective reading does not admit of this possibility but requires that the houses be owned jointly by John and Mary. The distinction between distributive and collective uses can be made explicit by adding each in the case of the distributive reading and together in the case of the collective reading.

The tree for the collective reading is:

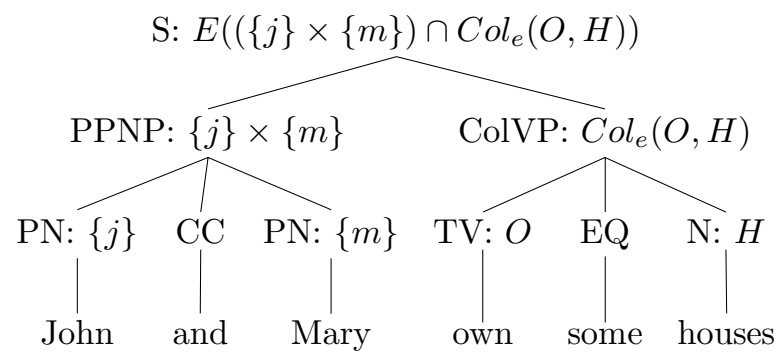

Recall from previous section that the distributive reading (3..4i $)$ is true if

$$
\{j, m\} \subseteq e(O, H) .
$$

This is equivalent to the condition

$$
(\exists u)(j O u \wedge u \in H) \wedge(\exists u)(m O u \wedge u \in H) .
$$

The collective reading is true if

$$
<j, m>\in O ; I_{H} ; \breve{O}
$$

This is equivalent to the condition

$$
(\exists z)(z \in H \wedge x O z \wedge y O z) .
$$

The collective reading is stronger than the distributive reading: $(4.8)$ is a logical

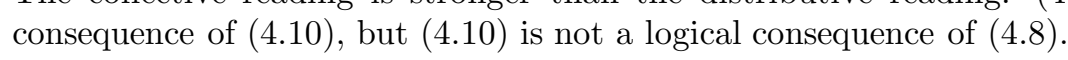

\section{Anaphoric Extension}

In this section our grammar is extended to derive semantic trees for constructions with anaphoric pronouns like each other, same and different. The rules are 17

$$
\begin{array}{llll}
\operatorname{ColV} P & \rightarrow T V+\operatorname{Rec} & {[\operatorname{ColVP}]} & =\operatorname{Rec}([T V]) \\
\operatorname{ColVP} \rightarrow T V+\operatorname{Rec}+\text { PossInf } & \rightarrow N & {[\operatorname{ColVP}]} & =\operatorname{RecPoss}([T V],[N]) \\
\operatorname{CplVP} \rightarrow T V+D A+I D+N & {[\operatorname{ColVP}]} & =\operatorname{Id}([T V],[N]) \\
\operatorname{ColV} P \rightarrow T V+D I V+N & {[\operatorname{ColVP}]} & =\operatorname{Div}([T V],[N])
\end{array}
$$

where lexical category abbreviations are as follows:

${ }^{17}$ The first rule corrects the analysis given in Böttner (1992b). 
186 A Collective Extension of Relational Grammar

$\begin{array}{lll}\text { ID } & \text { identity pronoun } & \text { same } \\ \text { DIV } & \text { diversity pronoun } & \text { different } \\ \text { DA } & \text { definite article } & \text { the } \\ \text { Rec } & \text { reciprocal pronoun } & \text { each other, one another }\end{array}$

Our grammar derives the following semantic tree:

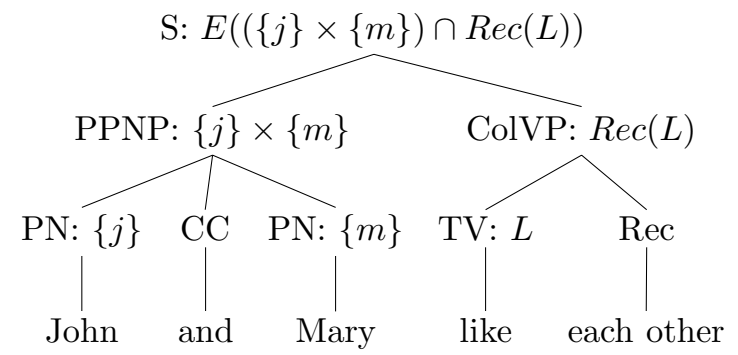

Notice that it is necessary to have the verb phrase denote $\operatorname{Rec}(R)=\operatorname{sym}(R)-I$ rather than $\operatorname{sym}(R)$. The reason for this is as follows: in a model structure with a singleton denoted by students and a singleton denoted by like like this

$$
\begin{array}{ll}
v(\text { students }) & =\{j\} \\
v(\text { like }) & =\{<j, j>\}
\end{array}
$$

the sentence All students like each other would be predicted to be true, since

$$
[\text { like each other }]=\operatorname{sym}([\text { like }])=\{<j, j>\} .
$$

But intuitively, we would not be willing to call this sentence true in such a situation, for we prefer to view like each other to involve at least two different individuals. Under the assumption

$$
[\text { like each other }]=\operatorname{sym}([\text { like }])-I=\emptyset
$$

the above sentence will be predicted to come out false.

For the sentence

$$
\text { All students like each other }
$$

our grammar derives the set-theoretical structure

$$
S \times S-I \subseteq \operatorname{Rec}(L) .
$$

$(5.3 i)$ amounts to the following

$$
(\forall x)(\forall y)(x \in S \wedge y \in S \wedge x \neq y \rightarrow x L y \wedge y L x \wedge x \neq y) .
$$

According to Langendoen's notion of strong reciprocity, 18 (1) $(5.2)$ case the following condition holds:

$$
(\forall x)(\forall y)(x \in S \wedge y \in S \wedge x \neq y \rightarrow x L z) .
$$

\footnotetext{
${ }^{18}$ Langendoen (1978).
} 
Although our condition (15.4) may look more complicated in comparison with Langendoen's condition ( $(5.5)$, the two conditions are in fact equivalent. Firstly, it is obvious that our condition implies Langendoen's condition, since both conditions have the same antecedents and Langendoen's succedent occurs as a constituent of the succedent of our condition. Secondly, our condition follows from Langendoen's condition, since

$$
<x, y>\in s \times S \leftrightarrow<y, x>\in S \times S
$$

from which both $x R y$ and $x R y$ follows.

For the sentence

$$
\text { Some students like each other }
$$

our grammar derives the set-theoretical structure

$$
E((S \times S) \cap \operatorname{Rec}(L))
$$

which is true just in case

$$
(\exists x)(\exists y)(x \in S \wedge y \in S \wedge x \neq y \wedge x L y \wedge y L x) .
$$

We think (5.8) to be in line with our intuition about the meaning of (5.6i).

In contradistinction to the truth-condition for (5.4), no corresponding condition can be derived for (5i) from Langendoen's definition of strong reciprocity. Bearing in mind the equivalence

$$
(\exists x) \alpha=\neg(\forall x) \neg \alpha
$$

one could derive the following condition for particular affirmative sentences from Langendoen:

$$
\neg(\forall x) \neg(\forall y)(x \in S \wedge y \in S \wedge x \neq y \rightarrow x L y)
$$

which is equivalent to

$$
(\exists x)(\forall y)(x \in S \wedge y \in S \wedge x \neq y \rightarrow x L y) .
$$

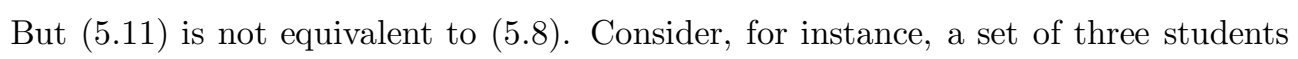
$a, b$, and $c$ with a relation $L$ (represented by the arrow) holding between them:

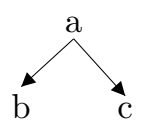

Then $(5 . \overline{1} \overline{1})$ would be true with respect to this model, since $a$ is the element that fulfils the condition, but (5.8in $y$ such that $x L y$ and $y L x$. On the other hand, the following model

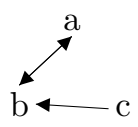

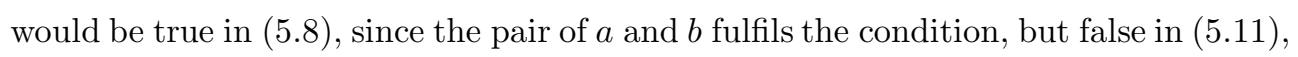
since there is no element from which every other element can "reached". So Langendoen's definition fails to account for particular sentences. Langendoen's definition suffers from being tailored for universal sentences. 
The grammar predicts the following inferences to be valid:

$$
\begin{aligned}
& \frac{\text { John and Mary like each other }}{\text { Mary and John like each other }} \\
& \frac{\text { John and Mary like each other }}{\text { John likes Mary }}
\end{aligned}
$$

The proof for $(1.12)$ follows from the fact that the relation denoted by the verb phrase like each other is symmetrical according to theorem 5:

$$
(A \times B)^{\cup} \cap R=(B \times A) \cap R
$$

Our grammar derives the following semantic tree for combinations of possessive and reciprocal:

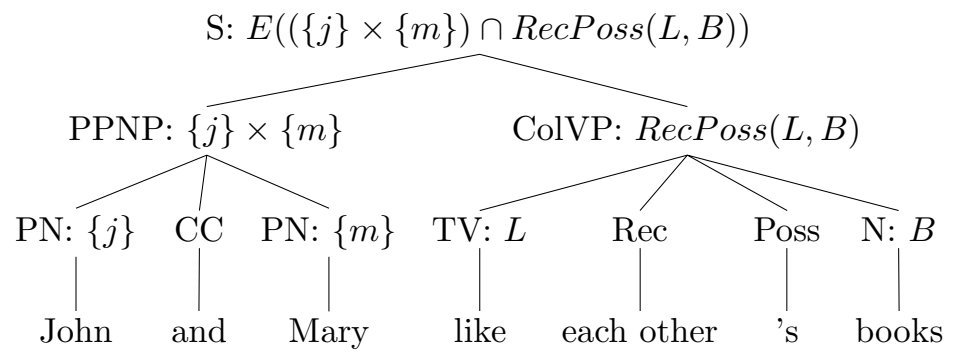

According to our grammar, the verb phrase like each other's books denotes the following relation:

$$
-\left(-L ; I_{B} ; \breve{P}\right) \cap-\left(P ; I_{B} ;-\breve{L}\right) \cap-I \text {. }
$$

And this is what it is supposed to denote, since

$$
<x, y>\in-\left(-L ; I_{B} ; \breve{P}\right) \cap-\left(P ; I_{B} ;-\breve{-L}\right)
$$

is equivalent to

$$
(\forall z)(z \in B \rightarrow((y P z \rightarrow x L z) \wedge(x P z \rightarrow y L x)))
$$

Our grammar predicts the following inference to be valid:

$$
\text { John and Mary like each other's books }
$$

The proof follows from Theorem 8 .

Notice that the sentence

$$
\text { John and Mary like each other's books }
$$

is true if the denotation of books is the empty set for the same reason why universal sentences are true if the subject term denotes the empty set, which is in line with the convention of standard interpretation. 
Contrary to our earlier pessimistic remarks, 19 constructions of the kind John and Mary like the same books can be accommodated within a relational algebra. This is by no means surprising since any partition is equivalent to some equivalence relation. That the relation denoted by read the same books is indeed an equivalence relation on the set of book readers follows from Theorem $2 . \overline{1} \overline{0}$.

Our grammar derives the following semantic tree:

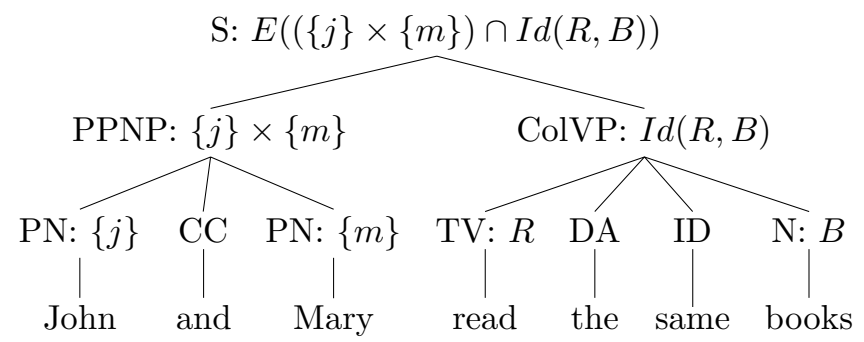

That $\operatorname{Id}(R, B)$ captures the intended meaning of the verb phrase is shown by being equivalent to the following quantifier logic expression:

$$
\{<x, y>\mid(\forall z)(z \in B \rightarrow(x R z \leftrightarrow y R z))\}
$$

Our grammar predicts the following inference to be valid:

\begin{tabular}{l} 
John and Mary read the same books \\
Bill and Mary read the same books \\
\hline John and Bill read the same books
\end{tabular}

The proof follows from Theorem 10 .

A parallel case is the verb phrase read different books. According to our grammar it denotes $\operatorname{Div}(R, B)$. That $\operatorname{Div}(R, B)$ construes the meaning of this verb phrase is shown by its equivalence to the following quantifier logic expression:

$$
\{<x, y>\mid(\forall z)(z \in B \rightarrow(x R z \leftrightarrow \neg y R z)\}
$$

There is an interesting duality between universal and negative quantifiers and same and different:

THEOREM 5.1

$U Q+N+T V+D I V+N \leftrightarrow N Q+N+T V+D A+I D+N$

Proof. From Theorem $2.122_{1}^{1}$ we have

1. $X \subseteq \operatorname{Div}(R, B) \rightarrow X \cap \operatorname{Id}(R, B)=\emptyset$

2. $X \cap \operatorname{Id}(R, B)=\emptyset \rightarrow X \subseteq \operatorname{Div}(R, B)$

THEOREM 5.2

$N Q+N+T V+D I V+N \leftrightarrow U Q+N+T V+D A+I D+N$

${ }^{19}$ Böttner (1992b). 
Proof. Analogous to proof of Theorem 5.1 .1$.

Theorem 5.1 and Theorem 5.21 predict the following pairs of sentences to be equivalent:

1.(a) All students read different novels

(b) No students read the same novels

2.(a) No students read different novels

(b) All students read the same novels

Our grammar predicts the following argument to be valid:

$$
\frac{\text { John and Mary own all houses together }}{\text { John and Mary own the same houses }}
$$

where by together we want to explicitly refer to the collective reading of this sentence. From theorem '2.14'

$$
\text { [own all houses together }] \subseteq[\text { own the same houses }]
$$

follows, from which the assertion follows.

The important point about the validity of $(15 . \overline{2} \overline{3})$ is that it supports our definition

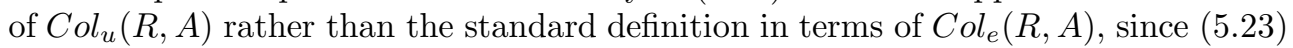
would not be valid with the weaker definition of this function.

Notice that our analysis of sentences

John and Mary like the same books

John and Mary like different books

preserves the verb phrases like the same books and like different books as constituents of the natural language sentences. Therefore we think this solution is more natural than the tree arising from the structure proposed by Carnap (1929) who proposed the sentence

Ich habe denselben Lehrer wie du

(I have the same teacher as you)

to have the logical form

$$
\breve{L} " i c h=\breve{L} " d u
$$

where $L$ is the denotation for Lehrer (teacher), ich and $d u$ are first and second person singular personal pronouns. Adopting Carnap's solution to our example would return

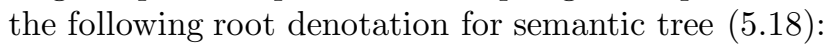

$$
B \cap R "\{j\}=B \cap R "\{m\} .
$$

This structure, however, does not match the syntactic structure of the respective sentence: Assume we let same denote the identity relation. We then have to deal with the fact that the constituent books occurs once in the syntactic structure but

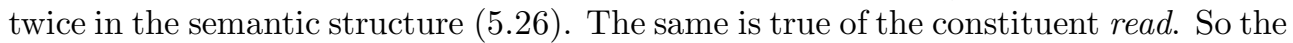
processing of the denotation would not be able to account for the subject-predicate structure of the sentence. Moreover, and what is by far worse, Carnap's solution cannot be generalized to

$$
\text { All students read the same books, }
$$


since its meaning requires a set of equations rather than a single equation:

$$
B \cap R "\left\{s_{1}\right\}=\ldots=B \cap R "\left\{s_{n}\right\} .
$$

We also think our solution to be more natural than the analysis in terms of polyadic quantifiers proposed in Keenan (1987) for the very same reasons as in the case of Carnap's analysis. Keenan's structure:

$$
\text { (ALL,SAME)(STUDENT,READ,BOOKS) }
$$

where the prefix is called a polyadic quantifier is too discrepant from the syntactic structure and fails to identify the verb phrase constituent as a predicate.

We assume that the verb phrase read the same books denotes a binary relation. Support comes from the fact that one can use it in a sentence like

$$
\text { John reads the same books as Mary. }
$$

And this sentence is clearly an instance of a relational sentence resembling comparative sentences like

$$
\text { John is as old as Mary. }
$$

\section{Conclusion}

Let us conclude by pointing out two open problems of our analysis that deserve further investigation. One problem has to do with the derivation of collective uses of transitive verbs, the other problem has to do with the extension of proper noun phrases from the combination of just two to more than two.

Our analysis predicts the following argument to be valid:

$$
\frac{\text { John and Mary own a house together }}{\text { John owns a house }}
$$

One might object against having this as a valid argument. But considering that collective ownership is

$$
\text { have a share in the possession of }
$$

rather than

$$
\text { share the possession of ... with }
$$

this inference may not be too devastating.

With our grammar so far we are not in a position to derive sentences with more than two proper noun combinations like

$$
\text { Tom, Dick, and Harry are brothers }
$$

So our grammar is in need for extension. A straightforward way to do this is by adding the rule-function-pair

$$
\begin{aligned}
P P N P & \rightarrow P N+C P N P \\
{[P P N P] } & =([P N] \cup[C P N P]) \times([P N] \cup[C P N P])
\end{aligned}
$$


It would derive the following semantic tree

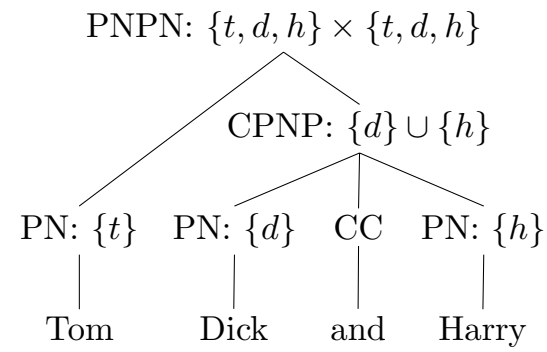

The sentence (6. 6.1$)$ would be true iff

$$
\{t, d, h\} \times\{t, d, h\} \cap B \neq \emptyset .
$$

This condition could be fulfilled if there is a pair $\langle x, y\rangle$ that belongs to both the denotations of Tom, Dick, and Harry and brothers, i.e. if, for instance, Tom is a brother of Harry. But this is not what (6.6) asserts. What it rather asserts is that any pair of the set of Tom, Dick, and Harry stands in the relation of being a brother of. This rules out either (6.5i) or the rule of grammar that accounts for expanding $S$ into PPNP + ColVP. Since we think (6.5) to be basically correct let us turn to the second alternative and replace the semantic function associated with the rule expanding $S$ and replace it by

$$
[S]=U(-[P P N P] \cup[C o l V P])
$$

This solution would at least return the desired result for $(6.4)$. But this solution runs into other problems. It would render any argument valid that has the form

$$
\frac{X+V P, Y \subseteq X}{Y+V P}
$$

But there are instances for which this form is not valid, like the following:

$$
\text { Tom, Dick, and Harry inhabit neighboring villages }
$$

\section{References}

[1] Blau, U. (1981). "Collective objects." Theoretical Linguistics, 8, 101-130.

[2] Böttner, M. (1992a). "State transition semantics." Theoretical Linguistics, 18, 239-286.

[3] Böttner, M. (1992b). "Variable-free semantics for anaphora." Journal of Philosophical Logic, 21, 375-390.

[4] Böttner, M. (1994). "Open problems in relational grammar." In Patrick Suppes: Scientific Philosopher, edited by P. Humphreys, 319-335. Kluwer.

[5] Brink, C. (1978). "On Peirce's notation for the logic of relatives." Transactions of the Charles S. Peirce Society, 14, 285-304.

[6] Brink, C. (1981). "Boolean modules." Journal of Algebra, 71, 291-313.

[7] Brink, C.; Britz, K.; and Schmidt, R. (1994). "Peirce algebras." Formal Aspects of Computing 6, 339-358.

[8] Carnap, R. (1929). Abriss der Logistik. Springer. 
[9] Hausser, R. (1974). "Syntax and semantics of plural." In Papers from the Tenth Regional Meeting of the Chicago Linguistic Society, edited by M. W. LaGaly, R. A. Fox, and A. Bruck, 234-247.

[10] Hoeksema, J. (1988). "The semantics of non-Boolean 'and'." Journal of Semantics, 6, 19-40.

[11] Keenan, E. L. (1987). "Unreducible n-ary quantifiers in natural language." In Generalized Quantifiers, edited by P. Gärdenfors, 109-150, Reidel.

[12] Keenan, E. L., and Faltz, L. M. (1985). Boolean Semantics for Natural Language. Reidel.

[13] Langendoen, (1978). "The logic of reciprocity." Linguistic Inquiry, 9, 177-197.

[14] Leonard, H. S., and Goodman, N. (1940). "Calculus of individuals and its uses." Journal of Symbolic Logic, 5, 45-55.

[15] Link, G. (1983). "The logical analysis of plurals and mass terms: a lattice-theoretical approach." In Meaning, Use, and Interpretation of Language, edited by R. Bäuerle, C. Schwarze, and A. von Stechow, 302-323, De Gruyter.

[16] Massey, G. J. (1976). "Tom, Dick, and Harry, and all the King's men." American Philosophical Quarterly, 13, 89-107.

[17] Peirce, C. S. (1880). "The logic of plural relatives." In Writings of Charles S. Peirce, edited by C. J. W. Kloesel, Vol. 4, (1986), 210-211, Indiana University Press.

[18] Scha, R. (1984). "Distributive, collective and cumulative quantification." In Truth, Interpretation and Information, edited by J. Groenendijk, T. M. V. Janssen, and M. Stokhof, 131-158, Foris.

[19] Suppes, P. (1960). Axiomatic Set Theory. Van Nostrand.

[20] Suppes, P. (1973). "Semantics of context-free fragments of natural languages." In Approaches to Natural Languages, edited by J. Hintikka, J. M. E. Moravcsik, and P. Suppes, 370-394, Reidel.

[21] Suppes, P. (1976). "Elimination of quantifiers by use of extended relation algebras." Revue Internationale de Philosophie, 30, 243-259.

[22] Suppes, P. (1979). "Variable-free semantics for negations with prosodic variation." In: Essays in Honor of Jaakko Hintikka, edited by R. Hilpinen, I. Niiniluoto, and M. P. Hintikka, 49-59, Reidel.

[23] Suppes, P. (1982). "Variable-free semantics with remarks on procedural extensions." In Language, Mind and Brain, edited by T. W. Simon, and R. J. Scholes, 21-34, Erlbaum.

[24] Suppes, P., and Macken, E. (1978). "Steps toward a variable-free semantics of attributive adjectives, possessives, and intensifying adverbs." In Children's language, edited by K. E. Nelson, Vol. I, 81-115, Gardner Press.

[25] Tarski, A. (1941). "On the calculus of relations." Journal of Symbolic Logic 6, 73-89.

[26] Tarski, A., and Givant, S. (1987). A Formalization of Set Theory without Variables. AMS colloquium Publications 41, Providence RI.

Received 14 February 1996 